\title{
Scope and Challenges of Internet of Things (IoT) in Kashmir.
}

\author{
Pala, M. Maqbool \\ J\&K Higher Education Department
}

\begin{abstract}
:
Internet of things is a fast growing technology that shall be shaping overall living standards of human beings all over the world. It, however like all other technological advancements, is dependent on certain factors. This paper tries to evaluate the scope, chances of growth and challenges that can hinder its survival in Kashmir.
\end{abstract}

Key Words: IOT, RFID, IP Address, WPAN, Embedded System, JRL, Curbs,ZigBee

\section{Introduction}

Wikipedia says

Quote:

The Internet of Things (IoT) is the interconnection of uniquely identifiable embedded computing devices within the existing Internet infrastructure.

So Internet of Things or IoT basically is connecting Embedded System to internet.

Succinctly: "The idea behind is that a whole collection of inanimate objects is being designed with built-in wireless connectivity, so that they can be monitored, controlled and linked over the Internet via a mobile app."

The types of objects range from wearables to light bulbs to home appliances (like the coffee maker, washing machine, and even your car) - really, anything. IoT is also being applied to vertical markets like the medical and health-care industry and Environment and Manufacturing Industry to transportation systems. Power supply to appliances can be turned off remotely using Mobile Apps.

These smart devices need to be connected to the Internet so that they share data and are remotely controlled. The various wireless radios that allow these devices to connect to the Internet and to each other include more familiar standards like Wi-Fi, low-energy Bluetooth, NFC and RFID, and some

less popular technologies, like ZigBee, $\underline{\mathrm{Z}}$ Wave and 6LoWPAN. These devices need to be uniquely identifiable on the network. IP addresses are allocated to these devices for that purpose. Since the quantity of devices is so huge that IPv4 cannot cater to this requirement, IPv6 is used instead.

According to McKinsey: "Sensors and actuators embedded in physical objects are linked through wired and wireless networks, often using the same Internet Protocol (IP) that connects the Internet".

The idea is to go beyond our computer and Smartphone and to make all other things around us share data and to provide

human beings with better and fast services that were never available before. These services can be of any nature available at anywhere and that too at any time. It is further to be determined the importance of Peace and some other pre-requisites for the success of this technology.

\subsection{Brief history of IoT}

The term Internet of Things is 16 years old. But the actual idea of connected devices had been around longer, at least since the 70s. Back then, the idea was often called "embedded internet" or "pervasive computing". But the actual term "Internet of Things" was coined by Kevin Ashton in 1999 during his work at Procter \& Gamble. Ashton who was working in supply chain optimization, wanted to attract senior management's attention to a new exciting technology called RFID. Because the internet was the hottest new trend in 1999 and 
because it somehow made sense, he called his presentation "Internet of Things".

\section{IoT compared to similar concepts}

Though the Internet of Things(IoT) is by far the most popular term to describe the organization of a connected world, there are some other technologies that are more or less similar. Most of these concepts are similar in meaning but they all have slightly different definitions.

\section{M2M}

The term Machine to Machine (M2M) has been in use for more quite long now, and is well-known in the Telecoms sector. M2M communication had been a one-to-one connection. But mobile connectivity gave the data sharing a different dimension wherein data can flow over a network to multiple destinations simultaneously.

\section{Industrial Internet (of Things)}

The term goes beyond M2M since it not only focuses on connections between machines but also includes human interfaces.

\section{Internet of Things (IoT)}

IoT has yet a wider reach as it also includes connections beyond the industrial context such as wearable devices on people.

\section{Web of Things}

The Web of Things is much narrower in scope as it focuses mainly on software architecture.

\section{Internet of Everything (IoE)}

Still not so popular, IoE aims to include all sorts of connections that one can think of. The concept has thus the highest reach.

\section{IoT applications}

There is hardly any area or walk of life where IoT can not be used or applied. Ranging from wearables to healthcare to space Missions, means every where and with every thing.

Today's major applications include:

\section{Smart home}

We can "automate" our homes using Smart Appliances, Smart TVs, Smart Washing Machines, Smart Surveillance system, Smart Doors and Smart Locks etc. It can also include thermostats, smoke detectors, lightbulbs as well. These wi-fi enabled devices can be made a part of Network and, hence, can be controlled remotely.

\section{Wearables}

Fitbit Flex, or the Apple Smartwatch are some of the wearables that make up a large part of the consumer facing Internet of Things applications.

\section{Smart City}

Smart city covers a wide variety of use cases like traffic management, Pure water distribution, waste management, urban security using Surveillance Cameras, street lights, Smart Parking etc.

\section{Smart grids}

A future smart grid promises to use information about the behaviors of electricity suppliers and consumers in an automated fashion to improve the efficiency, reliability, and economics of electricity. For example, Grids can provide real-time information about the dynamics of energy supply and consumption, helping in delivery of efficient and sustained electricity. Smart bulbs can be provided to the consumers which can turn off automatically when minimum use is sensed. Today's grid is very reliable and can deal with normal electricity fluctuations and it will take a step further towards using a low carbon energy system, by allowing integration between the renewable energy and green technologies, and offering many benefits to customer in cost savings through efficient energy use at home.

\section{Connected car}

Companies are in race to manufacture such a car that whether self-driving or just driver-assisted be capable of being connected with other cars. Other potentials like, mapping services, or traffic control, in-car entertainment systems and remote monitoring will also play a part

\section{Connected Health (Telehealth/Telemedicine)}

The concept of a wi-fi enabled and connected health care system and smart medical devices bears enormous potential, not just for manufacturers but also for the well-being of people in general: New kinds of Smart and connected health monitoring and improved medical decision-making based on large sets of patient data are some of the envisioned benefits. 


\section{Smart retail}

Data on Consumer behavior and seasonal requirements have been of great help to the retailers.

\section{Smart farming}

The remoteness of farming operations like Weather Forecasting, Controlled Irrigation, Monitoring of crop nutrients and monitoring of live stock makes farming an interesting case for the Internet of Things.

\section{Smart Environment}

Sensors can be placed at several areas in both Urban and Rural, Forests, near Water bodies to collect information regarding the type and level of pollution. The data can then be transmitted to relevant Control Centre for necessary action and measures.

\section{Scope of IoT in Kashmir}

Kashmir is a province of J\&K Sate in India. J\&K as a state has the fifth highest rate of per Capita Income in the country. Kashmir as a province is far ahead than other two provinces of the state. People spend lavishly on Home appliances and other smart things. Ratio of youth population to the number of Smart Phones has been found the highest in Kashmir than any other part of the country. In short, the chances of growth of Smart things in Kashmir is huge. However, there are certain challenges that need to be addressed at the earliest so that people in this part of the globe reap the benefits of this soul soothing technology.

\section{Challenges}

\subsection{Technical}

The applications above look very attractive; but nothing comes free; it faces equally hard and difficult challenges. Besides, the need for efforts to make these smart things available at a lower price so that the benefit reaches to the maximum, we are also faced with many other challenges, such as:

Scalability: An Internet of Things potentially has a larger overall scope than the conventional Internet of computers. But then again, things cooperate mainly within a local scope. So, the operational capability needs to be enhanced to cover large-scale environments.

"Arrive and operate": Smart objects should not be taken as computers that require their users to configure and adapt them to particular situations. Mobile things, which are often only thinly used, need to establish connections spontaneously, and organize and configure themselves to suit a particular environment.

Interoperability: Since the world of physical things is extremely diverse, in an Internet of Things each type of smart object is expected to be connected with a network. We can make a smart device talk to another device if and only if both the devices follow some sort of compatibility. Since different organizations follow different standards, it is very difficult to make these devices communicate. ISO must intervene to find solutions on the lines of IP standards.

Discovery: In dynamic environments, suitable services for things must be automatically identified, which requires appropriate procedures of describing their functionality. Users must receive productrelated information, and should use search engines to find things or provide information about an object's state.

Software complexity: Like embedded systems, these smart objects or things must be supported by extensive software infrastructure and background servers to manage the devices and provide services to them.

Data volumes: volume of data and its frequency used by some application scenarios might vary in certain cases, the devices must be capable enough to communicate at different frequencies with different volumes of data with the central nodes.

Data interpretation: To support the users of smart things, efforts would be to interpret the local context determined by sensors as accurately as possible. For service providers to profit from the diverse data that will be generated from diverse sources with diverse formats, it would need to be able to draw some generalizable conclusions from the interpreted sensor data. Big Data Analytics can be used along with data warehousing to solve this problem.

Security and personal privacy: In addition to the security issues of the Internet which we all are familiar with (such as communications confidentiality, the authenticity and trustworthiness of communication partners, and message integrity), other requirements would also be important in an Internet of Things. We might want to give things only selective access to certain services, or prevent them from communicating with other things 
unnecessarily or in an uncontrolled manner as such business transactions involving smart objects would mean compromising with the business future.

Fault tolerance: Internet of things is, undoubtedly, large in scale and functionality than Internet of Computers and, hence, the dependence will be high. So, Redundancy on several levels would become paramount.

\section{Social and Political}

Power supply: smartness of these smart devices needs to be powered from a self-sufficient energy source. Though some passive RFID transponders do not need their own energy source, their functionality and communications range are very limited. In many scenarios, batteries and power packs are problematic due to their size and weight, and especially because of their maintenance requirements.

In Kashmir, where power is generated to cater to $40 \%$ of energy requirements of India, the load shedding or power curtailments are so frequent and for big gaps. The curtailments are not even defined. The idea of having Internet of Things in Kashmir looks very distant a dream at-least in such scenario. Now, one can think of using battery and power packs instead, but these batteries also require power to be refreshed. And the battery technology is making relatively slow progress, generating electricity from the environment (using temperature differences, vibrations, air currents, light, etc.), is not yet powerful enough to meet the energy requirements of current electronic systems in many application scenarios. Hopes are pinned on future low-power processors and communications units for embedded systems that can function with significantly less energy.

Wireless communications: From an energy point of view, established wireless technologies such as GSM, Wi-Fi and Bluetooth are far less suitable; more recent WPAN standards such as ZigBee and others still under development may have a narrower bandwidth, but they do use significantly less power.

Un-Interruptible Connectivity: Smartness of these smart devices is worth going for only after they are

[1] Saranya C. M., Nitha K. P., Analysis of Security methods in Internet of Things. International Journal on Recent and Innovation Trends in Computing and Communication, Volume 3, Issue 4; April 2015.

[2] Knud Lasse Leuth; IOT Basics: Getting started with the Internet of Things connected to some network where they not only can share data but also can be controlled, monitored and managed remotely.

In Kashmir due certain circumstances, government puts curbs on Internet as a tool for law and order. The frequency of such curbs is, unavoidably, very high. These devices can never serve their purpose in such situations and, hence, the chances of such a comfortable technology growing in Kashmir in near future look bleak. Frequent strike calls given by Joint Resistance Leadership (JRL) forces government to go for Internet shutdown to avoid any untoward incident. So, restoration of normalcy is of paramount importance for making this technology make its inroads in the valley of Kashmir.

\section{Conclusion}

Internet of things, a rather new technology, provides many applications to connect the things to things, machine to machine, and human to things through the Internet. Each object (thing) in the world is uniquely identifiable. These objects can be made to communicate with each other through a central node, can be monitored and controlled remotely. Other networks and technologies used in building this concept of Internet of things are mobile computing, RFID, wireless sensor networks, and embedded systems, in addition to many algorithms and software infrastructure for management processes, storing data, and security issues. Standardization on the lines of IP Addresses is required. Big data analytics is needed to take care of standardization of interpretation of data generated at diverse sources with different formats and different sizes of data sets.

Among other issues, are security functions such as authentication and encryption, and functions to deliver voice and video signals efficiently; but in coming times these issues can be taken care of as several multinational companies are joining hands for the matter. The author pledges to share his comments with concerned quarters in the mainstream and in civil society for better future of this region.

\section{References}

[3] Friedemann Mattern and Christian Floerkemeier; From the Internet of Computers to the Internet of Thimgs.

[4] Grandinetti, Lucio. Pervasive Cloud Computing Technologies: Future Outlooks and Interdisciplinary Perspectives: Future Outlooks 
and Interdisciplinary Perspectives. IGI Global, 2013.

[5] Lopez Research; An Introduction to the Internet of Things.

[6] Economical Survey; GOI

[7] Mobile Users in different states by TRAI, GOI

[8] http://standardsinsight.com/iot/iotworkshop

[9] Debasis Bandyopadhyay, Jaydip Sen. Internet of Things - Applications and Challenges in Technology and Standardization. arvix 9 may 2011

[10] http://www.academia.edu/3276195/Internet_ of_Things_Applications_and_Challenges_in_Te chnology_and_Standardization

[11] Adam D. Thierer. The Internet of Things and Wearable Technology: Addressing Privacy and Security Concerns without Derailing Innovation. 21 Rich. J. L. \& Tech. 6 (2015).

[12] Patrick Guillemin et al., Internet of Things standardization - Status, Requirements, Initiatives and Organizations. Conference: Internet of Things - Converging Technologies for Smart Environments and Integrated Ecosystems 2013.

[13] Sapandeep Kaur, Ikvinderpal Singh. A Survey Report on Internet of Things Applications. International Journal of Computer Science Trends and Technology Volume 4, Issue 2, Mar - Apr 2016.

[14] Isam Ishaq et al., IETF Standardization in the Field of the Internet of Things (IoT): A Survey. J. Sens. Actuator Netw. 2 (2013) 235-287, doi: 10.3390/jsan2020235

[15] Nicola Accettura. Optimal and Secure Protocols in the IETF 6TiSCH communication. stack;

http://telematics.poliba.it/publications/2014/Acc ettura ISIE2014 .pdf

[16] Dr Ovidiu Vermesan, Dr Peter Friess. Internet of thing from research and innovation to market deployment, 2014 River Publishers

[17] http://www.standardsuniversity.org/emagazine/march-2016/security-and-iot-in-ieeestandards/

[18] Patrick Guillemin, et al., Internet of Things Position Paper on Standardization for IoT technologies. European research cluster on the internet of things; January, 2015.

[19] http://www.7wdata.be/article-general/howbig-data-and-internet-of-things-builds-smartcities/

[20] Gabriele Lobaccaro, Salvatore Carlucci and Erica Löfström (2016). A Review of systems and Technologies for Smart Homes and Smart Grids.

[21] Amrita Sajja, D. K. Kharde, Chandana Pandey. A Survey on efficient way to Live: Smart

Home - It's an Internet of Things. ISAR International Journal of Electronics and Communication Ethics, Volume 1. Issue 1, 2016.

[22] Eiman Al Nuaimi1 et al., Applications of big data to smart cities. Journal of Internet Services and Applications 2015.

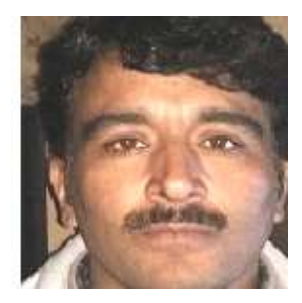

Pala, M. Maqbool did his Master of Computer Applications from Jammu University in 19961999. Worked as Information Officer Kashmir University 2000-2004. Since 2004, he is serving as Asst. Professor Higher Education J\&K SateIndia. 